\title{
AHP and Uncertainty Theories for Decision Making using the ER-MCDA Methodology
}

\section{Jean-Marc Tacnet \\ Cemagref-ETGR}

2, rue de la papèterie B.P. 76

38402 Saint Martin d'Heres Cedex, France.

Email: jean-marc.tacnet@cemagref.fr
Jean Dezert

ONERA - The French Aerospace Lab.

Chemin de la Hunière

91761 Palaiseau Cedex, France.

Email: jean.dezert@onera.fr

\author{
Mireille Batton-Hubert \\ EMSE - SITE \\ 29, rue Ponchardier \\ 42100 Saint-Etienne, France. \\ Email: batton@emse.fr
}

\begin{abstract}
In this paper, we present the $E R-M C D A$ methodology for multi-criteria decision-making based on imperfect information coming from more or less reliable and conflicting sources. The Analytic Hierarchy Process $(A H P)$, Fuzzy Sets, Possibility and Belief Functions theories are combined to take a decision based on imprecise and uncertain evaluations of quantitative, qualitative criteria. Classical aggregation of criteria is replaced by a two-step fusion process using advanced fusion rules based on the Dezert-Smarandache Theory (DSmT) that allows to make a difference between importance, reliability and uncertainty of information sources and contents.
\end{abstract}

Keywords: Analytic Hierarchy Process $(A H P)$, information imperfection, uncertainty, information fusion, fuzzy sets theory, possibility theory, belief function theory, Dempster-Shafer theory (DST), Dezert-Smarandache theory (DSmT), expert assessment, natural hazards.

\section{INTRODUCTION}

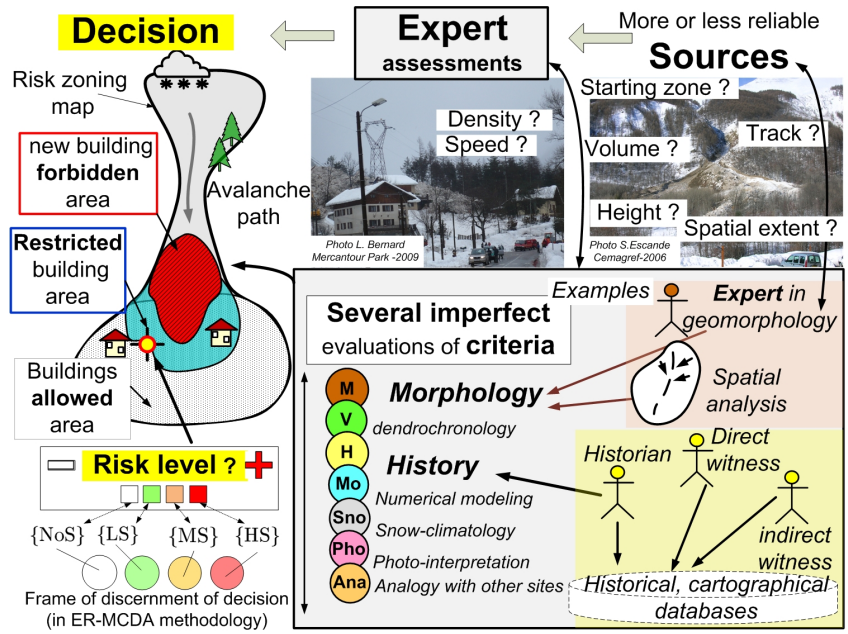

(a) Information, expert assessments and decision are linked

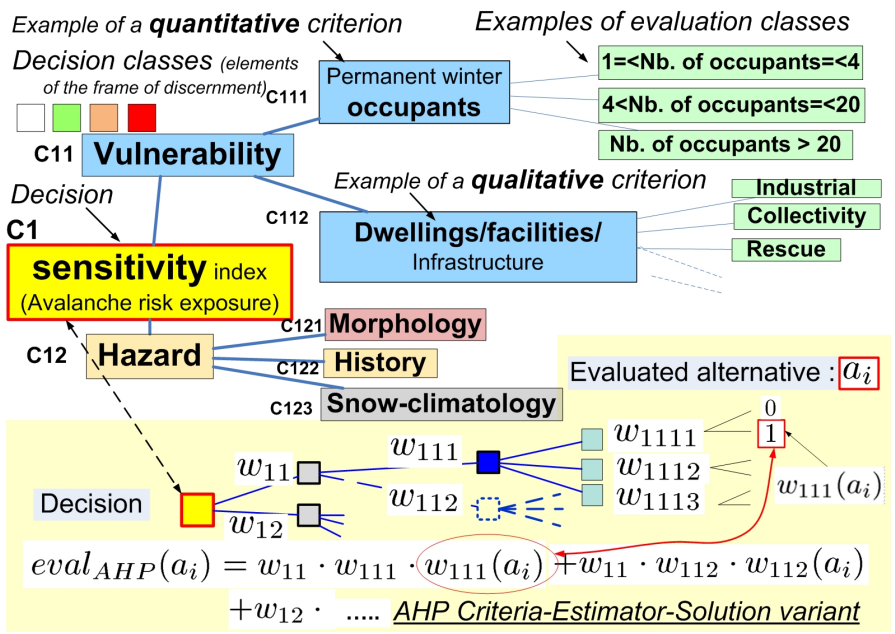

(b) Hierarchical decision modeling

Figure 1: decision problem related to avalanche risk zoning.

In many real-life contexts, decisions are based on multiple criteria with variable expressed by the decision-makers. Most of time, available information to evaluate those criteria remain imperfect and the sources of this information appear as more or less reliable and conflicting. The question is therefore to propose decision support methods that are able to consider both the complexity or real-life decision problems including relative and subjective importance of the criteria, the information imperfection and the heterogeneity, reliability and conflict level of information 
sources. In the context of natural hazards in mountains, an example of decision problem consists in choosing what is the snow avalanche risk level on a given avalanche path (figure 1). This decision results from different expert assessments provided on the basis of more or less reliable sources depending on thematic domains such as geomorphology, history, numerical modeling...(figure 1a). A simplified version of this decision problem [17] can be represented through a hierarchic model based on a variant of the original AHP method [11] based on a criteriaestimator-solution principle (figure 1b). Expert assessments are considered as a decision process based on imperfect information provided by more or less heterogeneous, reliable and conflicting sources. This article presents a new methodology called ER-MCDA designed to both help make decisions and also consider information imperfection represented by subjective, imprecise and uncertain qualitative and quantitative evaluations provided by several more or less reliable sources. First, we briefly review the existing theories to represent information imperfection and the way uncertainty has already been considered in multicriteria decision analysis. In a second part, the principles and main steps of the ER-MCDA methodology are described. The previous simplified version of risk zoning in the context of natural hazards management in mountains is used as a basic example for this methodology. Finally, the further developments and perspectives are discussed in the conclusion.

\section{INFORMATION IMPERFECTION AND UNCERTAINTY THEORIES}

Any decision is closely related to information quality. Uncertainty, often used in common language, is indeed only one of all the various types of information imperfection which are inconsistency, imprecision, incompleteness and uncertainty (figure 1b).

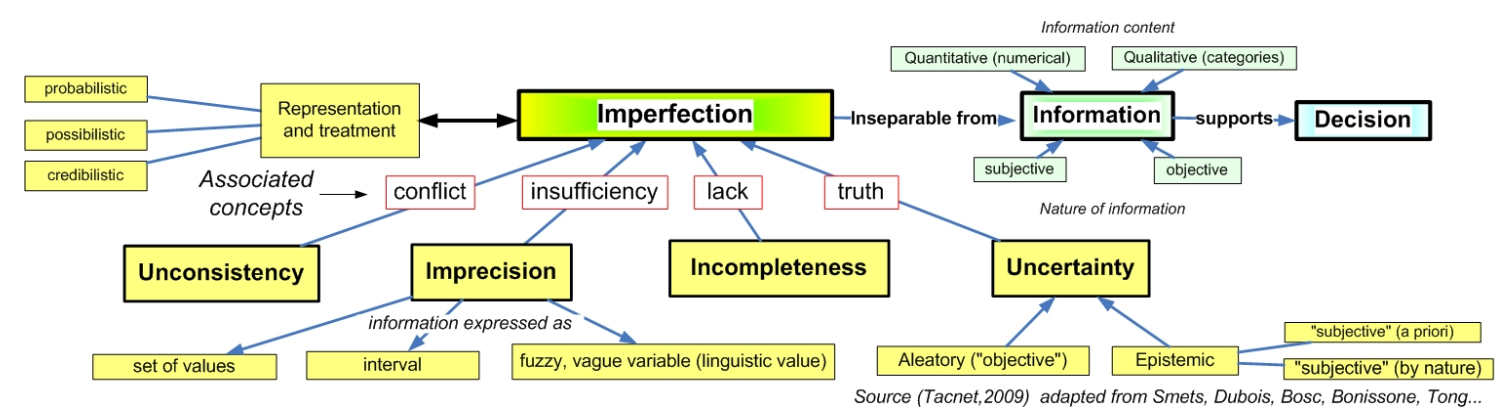

Figure 2: different kinds of information imperfection [18].

Probability theory is widely used to represent uncertainty but fails to handle vague, imprecise, uncertain and conflicting information. New theories have proposed to handle those different types of imperfect information.

\section{A. Fuzzy sets and possibility theories}

Fuzzy sets theory [23] represents vague information and relates an imprecise quantitative evaluation (e.g a number of occupants in figure 3a)with a qualitative category as defined by an expert (e.g. low, medium or high population density in figure 3a). Possibility theory [8], [24] represents both imprecision and uncertainty using possibility distribution. Instead of a single discrete evaluation, several consonant intervals with increasing confidence levels can be chosen: the wider the interval is, the more confident the expert is in his evaluation of the criterion (figure $3 b)$.

\section{B. Belief function theory}

Evidence or belief function theory allows one to represent and fuse information evaluation provided by more or less reliable and conflicting sources on the same hypotheses of a set called the frame of discernment. Each source (e.g. an expert) defines basic belief assignments (bba's). The hypotheses must be exhaustive and exclusive in the Dempster-Shafer (DST) framework [13], while the Dezert-Smarandache theory (DSmT) relaxes this hypothesis and provides a versatile framework to represent imprecise but also vague concepts (figure 4). All basis of $D S m T$ with many detailed examples can be obtained freely by downloading the three volumes given in [15] from the web. 


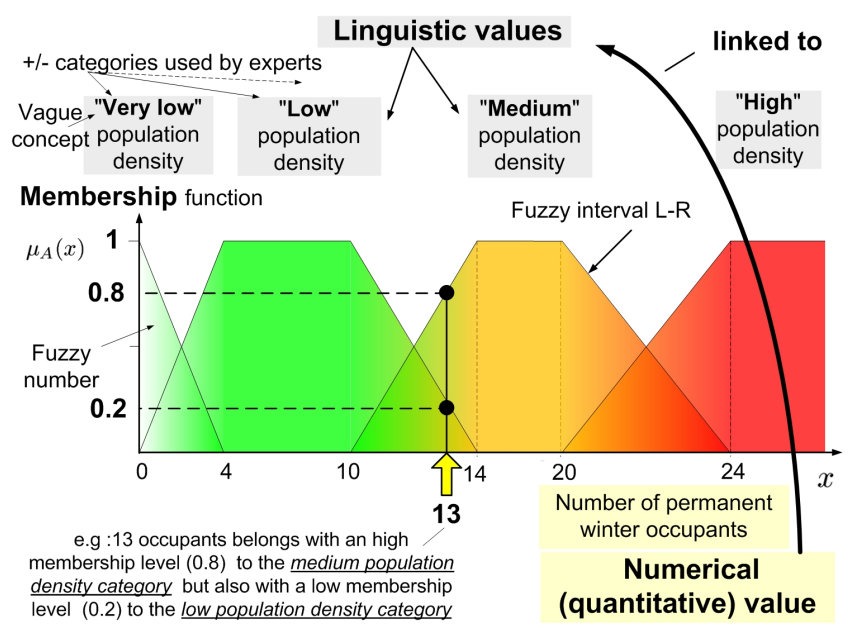

(a) Principles of fuzzy sets theory

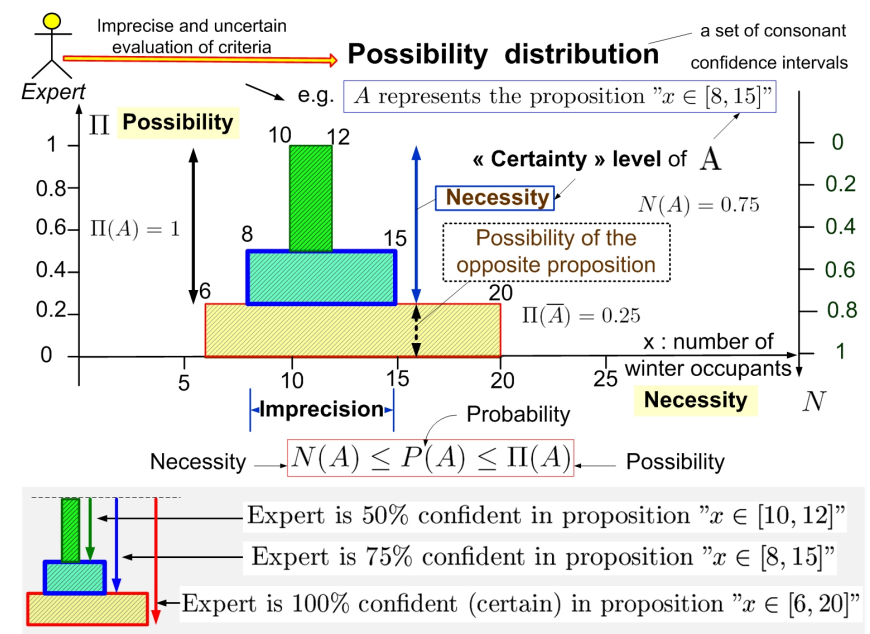

(b) Principles of possibility theory

Figure 3: representation of imprecise evaluation.

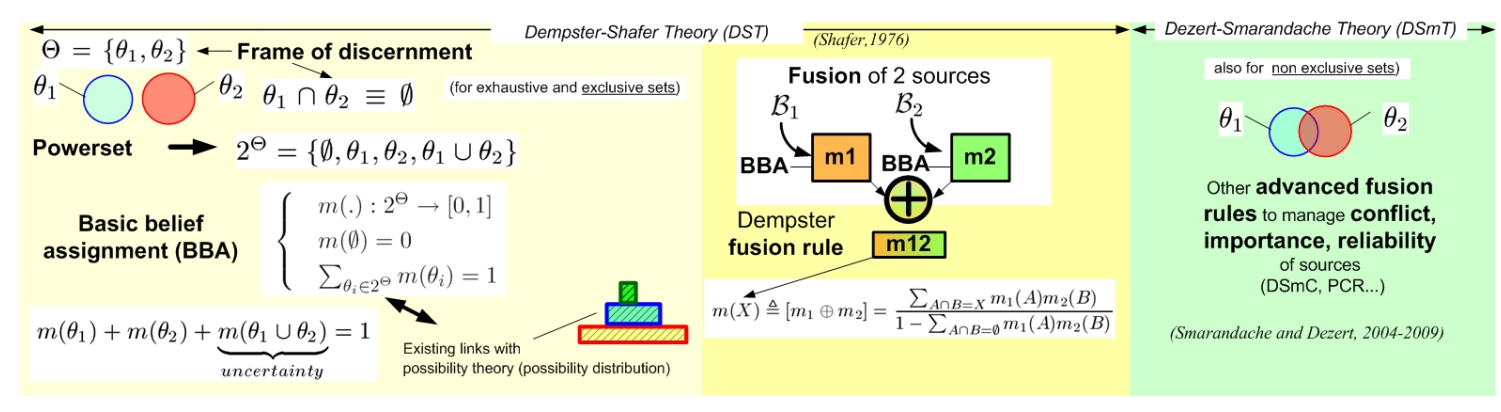

Figure 4: basics of Dempster-Shafer theory [13] and Dezert-Smarandache theory [15].

Information fusion consists in merging information stemming from several sources to answer questions or more generally to make decisions [4]. Sources can be discounted with regard to their reliability. Specific discounting methods have recently been proposed to consider separately importance and reliability in the context of multicriteria decision making [6], [16], [17]. Uncertainty and imprecision in multi-criteria decision models remains an important issue [14]. MAUT in general [22] and $A H P$ in particular have already been associated to the Evidence Theory [2] including the cases of several sources [?]. We propose now a new methodology able to consider both information imperfection, source reliability, importance and conflict and using the fuzzy sets, possibility and belief function theories.

\section{THE ER-MCDA METHODOLOGY}

This new framework called ER-MCDA mixes a multi-criteria decision making method called Analytic Hierarchy Process $(A H P)$ and uncertainty theories including fuzzy sets, possibility and belief function theories [17]. The principle of the $E R-M C D A$ methodology is to use $A H P$ to analyze the decision problem and to replace the aggregation step by two successive fusion processes [19]. Its main objective is to take into account both information imperfection, source reliability and conflict.

\section{A. Main principles}

The Analytic Hierarchy Process (AHP) [11], [12] is a single synthesizing criterion approach. Possible alternatives are evaluated according to preferences (represented by weights $w_{j}$ ) expressed by the decision-makers on the different criteria $\left(C_{j}\right)$ (figure 1b). Its principle is to arrange the criteria considered as important for a decision in a hierarchic 
structure descending from an overall goal to criteria, sub-criteria and finally alternatives in successive levels. It is based on three basic steps: decomposition of the problem, comparative judgments and hierarchic composition or synthesis of priorities. The ER-MCDA methodology uses the conceptual framework of $A H P$ both to analyze the decision problem (representing it through a weighted hierarchy of criteria and sub-criteria) and to identify preferences between criteria. The ER-MCDA introduces several new features. Its main steps (described below on figure 5a) consist in (1) analyzing the decision problem through a hierarchical structure, (2) defining the evaluation classes for decision through a common frame of discernment, (3) evaluating the qualitative or quantitative criteria, (4) mapping the evaluations of criteria into the common frame of discernment for decision, (5) fusing the mapped evaluations of criteria to get a basic belief assignment related to the evaluations classes of decision (frame of discernment for decision). These steps are independent one from each other. Therefore, imprecise and uncertain evaluations of quantitative or qualitative criteria can be done by the sources (experts) and re-used with different mapping models. We use a sample decision model developed to assess the sensitivity of a snow avalanche site according to the main criteria denoted as hazard (morphology, history and snow climatology) and vulnerability (winter occupants, dwellings and infrastructures). The decision consists in choosing a sensitivity level (no, low, medium, high sensitivity) (figure 1b). Those levels are the decision hypotheses that are used to define the common frame of discernment for information fusion.

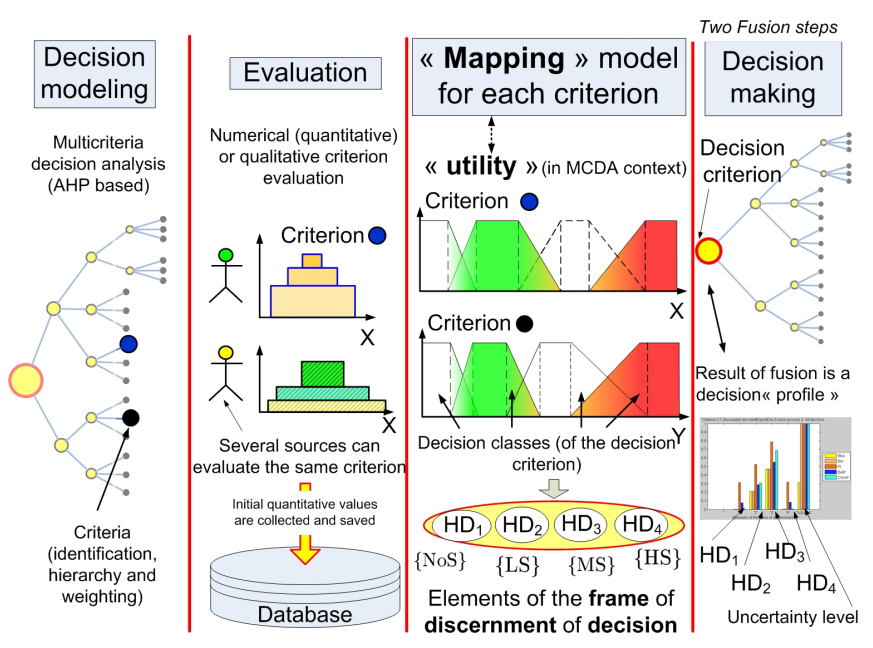

(a) Dissociation of four steps

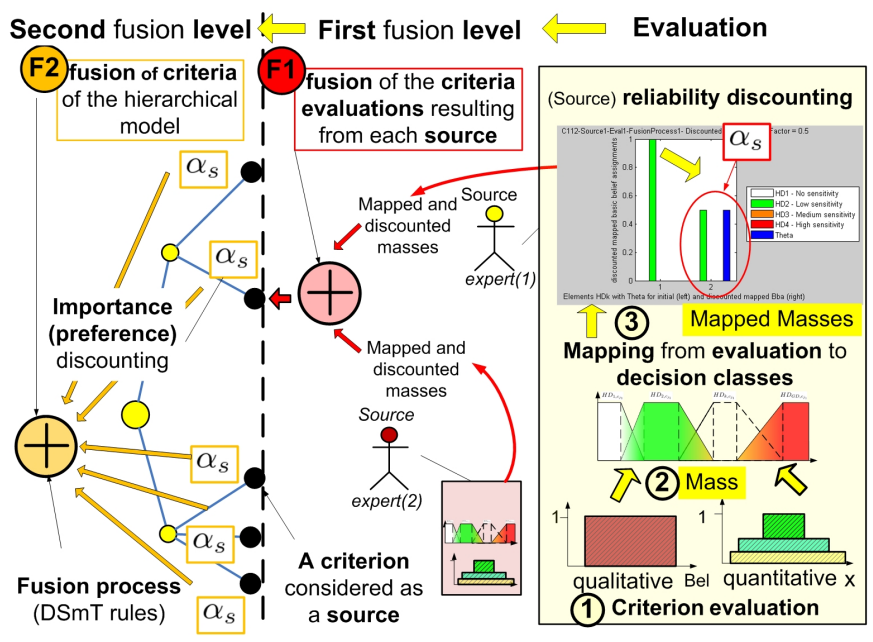

(b) Two steps of fusion are combined

Figure 5: decision problem related to avalanche risk zoning.

\section{B. Imprecise evaluation and mapping of criteria}

Quantitative criteria, such as the number of occupants (figures $1 \mathrm{~b}$ and $6 \mathrm{a}$ ), are evaluated through possibility distributions representing both imprecision and uncertainty. The source (an expert) provides evaluations as intervals with confidence level: for criterion $C_{111}$, the expert has a $75 \%$ level of confidence that the number of occupants will be in interval $[8,15]$. The question is then to transform a number of occupants into a level of sensitivity keeping information about uncertainty. Possibility distribution can be derived into basic belief assignments (bba's) [1]. We use a mapping process that project the bba's expressed on intervals on bba's expressed on the frame of discernment of decision (low- $L S$, medium- $M S$ and high- $H S$ sensitivity levels). After this evaluation step (figure $5 \mathrm{~b}$ ), we get, for each criterion, bba's related to the same frame of discernment.

\section{Fusion of evaluations and criteria}

A first fusion process ( $F 1$ in figure $5 b$ ) is done for all evaluations of the different sources for a same criterion. The bba's can be discounted according to the reliability level of each source. We finally get bba's for each criteria whose weights (related to their importance) have been defined according to the classical AHP method. Those 


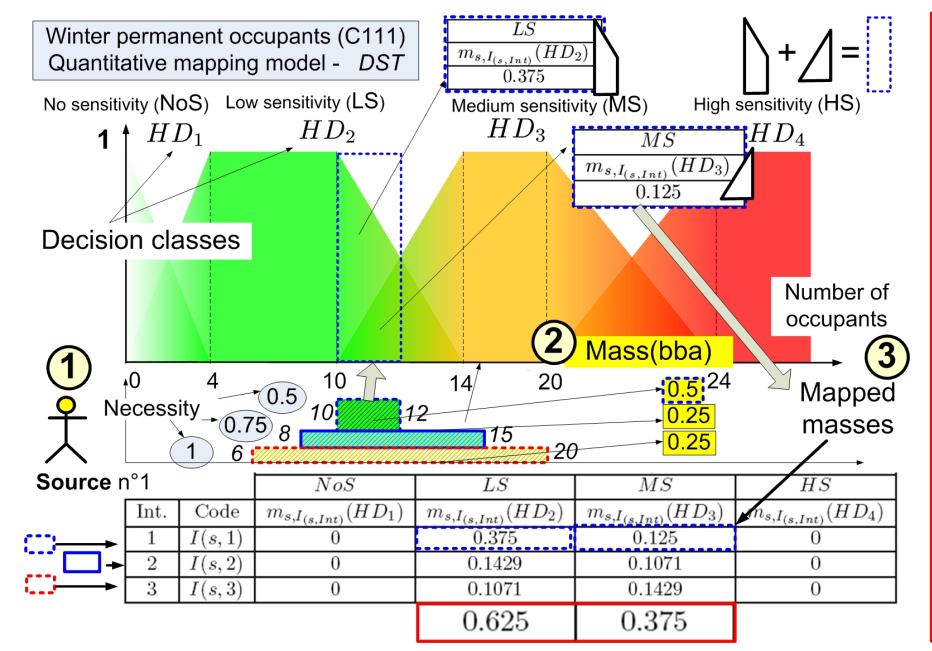

(a) Mapping process of a qualitatiive criterion

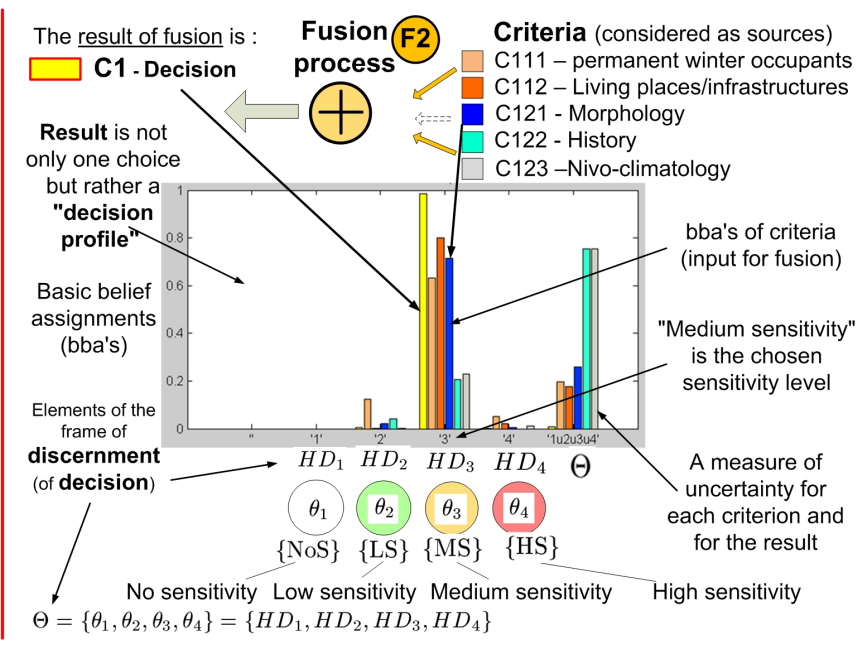

(b) Decision profile

Figure 6: mapping process: from evaluation to the frame of discernment of decision.

weights are derived into importance discounting factors (figure 7a. The bba's corresponding to each criterion (occupants, morphology ...) are then fused a second time ( $F 1$ in figure $5 \mathrm{~b}$ ) to get the final result which is called a decision profile. This profile shows not only the decision to take (here the $M S$ sensitivity level) but provides also an evaluation of the distribution of knowledge on the other levels and uncertainty. It is possible to check if all sources agree about the decision and also to have an idea about the uncertainty of their evaluation. The quality of information leading to decision is related to the decision itself. The results can be bba's or belief, plausibility values that correspond to pessimistic or optimistic choice of a decision level.

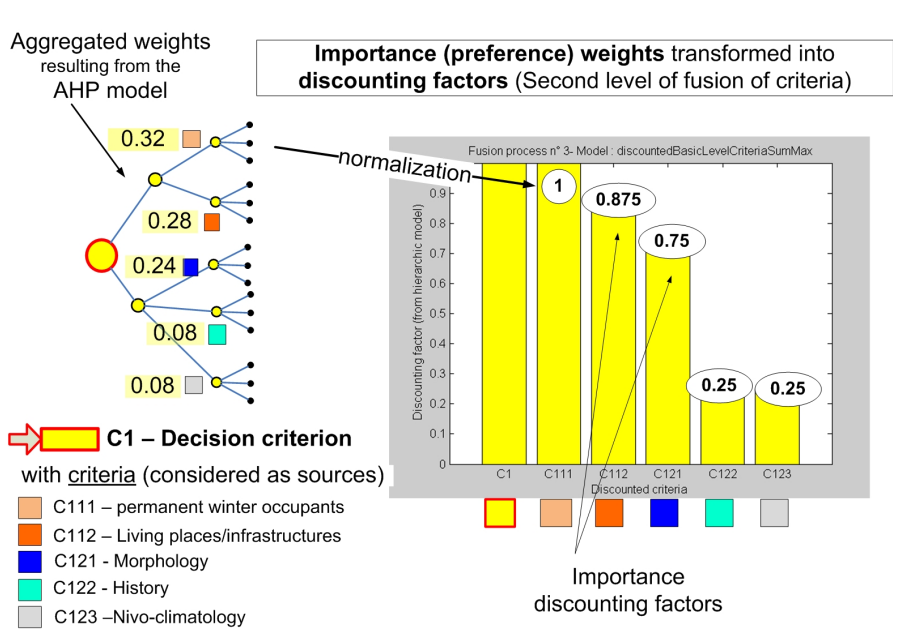

(a) From $A H P$ to importance discounting

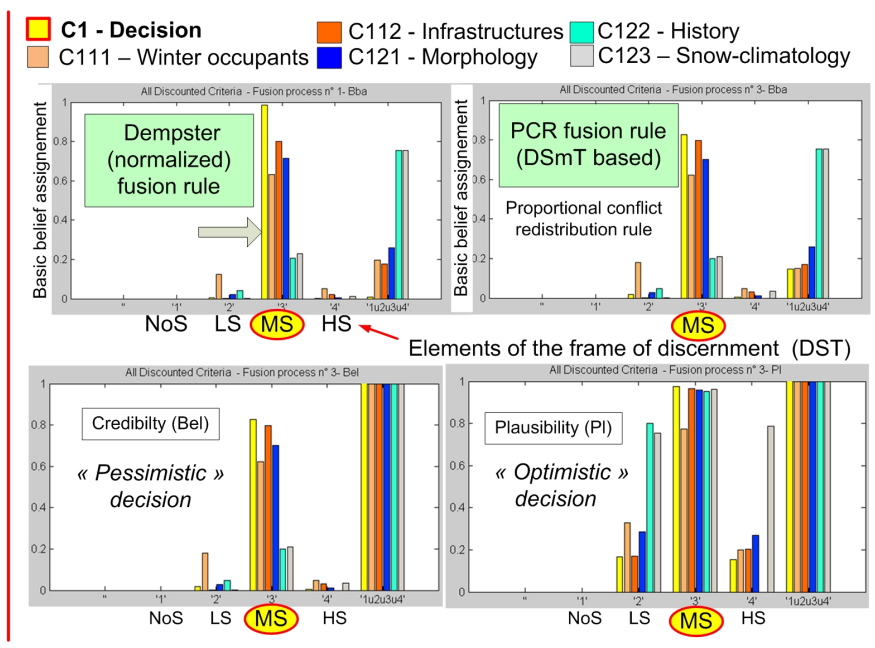

(b) Results

Figure 7: discounting and fusion results.

The ER-MCDA methodology uses specific fusion rules (figure 7b) such as the PCR fusion rules [5] [10] that have been developed in the context of the Dezert-Smarandache Theory ( $D S m T)$ to palliate the disadvantages of the classical Dempster fusion rule known to fail in high conflict situations. The importance of criteria is a different concept than the classical reliability concept developed and used in the belief theory context. In order to make a 
difference between importance of criteria, uncertainty related to the evaluations of criteria and reliability of the different sources, specific methods such as DSmT-AHP [6] have extended the Saaty's AHP method [11] using an aggregation method developed in Dezert-Smarandache Theory $(D S m T)$ framework [15] of evidential reasoning (ER).

\section{CONCLusions}

The originality of the ER-MCDA methodology consists in the association of different theories. Its main advantages are first its ability to dissociate imperfect evaluations from their combination in the fusion process and also the possibility to consider both evaluation imperfection and heterogeneity of sources.This methodology uses others developments for Multi-Criteria Decision-Making (MCDM) that are based on the combination of AHP method developed by Saaty and DSmT. The AHP method allows to build bba's from DM preferences of solutions which are established with respect to several criteria. The $D S m T$ allows to aggregate efficiently the (possibly highly conflicting) bba's based on each criterion. DSmT-AHP method also allows to take into account also the different importances of the criteria and/or of the different members of the decision-makers group [17], [6], [16], [7]. Developments of this methodology are still under progress. New theoretical methods are now proposed to deal with the case of decisions under uncertainty [21] and to extend the approach to spatial applications [20].

\section{REFERENCES}

[1] C. Baudrit, D. Guyonnet, and D. Dubois, Postprocessing the hybrid method for addressing uncertainty in risk assessments, Journal of Environmental Engineering,vol. 131, no. 12, pp. 1750-1754, 2005.

[2] M. Beynon, B. Curry, P.H. Morgan, The Dempster-Shafer theory of evidence: An alternative approach to multicriteria decision modelling, Omega, Vol. 28, No. 1, pp. 37-50, 2000.

[3] M. Beynon, A method of aggregation in ds/ahp for group decision-making with the non-equivalent importance of individuals in the group, Computers and Operations Research, 32:1881-1896, 2005.

[4] I. Bloch, A. Hunter and al.,Fusion: General concepts and characteristics, International Journal of Intelligent Systems,16(10):11071134,2001 .

[5] J. Dezert and F. Smarandache, Proportional Conflict Redistribution Rules for Information Fusion in [15], Vol.2, pp. 3-68, 2006.

[6] J. Dezert, J.-M. Tacnet, M. Batton-Hubert, F. Smarandache,Multi-criteria decision making based on DSmT-AHP in Proc. of Belief 2010 Int. Workshop, Brest, France, 1-2 April, 2010.

[7] J. Dezert, J.-M. Tacnet, Evidential Reasoning for Multi-Criteria Analysis based on DSmT-AHP, submitted to Proceedings of ISAHP 2011 Int. Conf., Sorrento, Italy, June 15-18th, 2011.

[8] D. Dubois and H. Prade, Possibility Theory:an approach to Computerized Processing of Uncertainty, Plenum Press, New York (U.S.A), 1988.

[9] J. Figueira J., S. Greco, M. Ehrgott (Eds.), Multiple Criteria Decision Analysis: state of the Art Surveys - vol. 78 of International Series in Operations Research and Management Science, Springer Verlag, Boston, Dordrecht, London,2005.

[10] A. Martin, Implementing general belief function framework with a practical codification for low complexity in [15], Vol.3, pp. 217-273, 2009.

[11] T. Saaty, The analytic hierarchy process, McGraw Hill, New York, 1980.

[12] T. Saaty, The analytic hierarchy and analytic network processes for the measurement of intangible criteria and for decision making in [9], pp.345-407, 2005.

[13] G. Shafer, A mathematical theory of evidence, Princeton University Press, 1976.

[14] T. J. Stewart, Dealing with uncertainties in MCDA in [9], pp.445-470, 2005.

[15] F. Smarandache, J. Dezert (Editors), Advances and Applications of DSmT for Information Fusion, American Research Press, Rehoboth, Vol.1-3, 2004-2009 - see http://fs.gallup.unm.edu//DSmT.htm.

[16] F. Smarandache, J. Dezert, J.-M. Tacnet, Fusion of sources of evidence with different importances and reliabilities, in Proceedings of Fusion 2010 Int. Conf., Edinburgh, UK, July 26-29th, 2010.

[17] J.-M. Tacnet, M. Batton-Hubert, J. Dezert, Information fusion for natural hazards in mountains in [15], Vol. 3, pp. 565-659, 2009.

[18] J.M. Tacnet, Prise en compte de l'incertitude dans l'expertise des risques naturels en montagne par analyse multicritères et fusion d'information, Phd in Environmental Engineering Sciences, Ecole Nationale Supérieure des Mines, Saint-Etienne, France, 2009.

[19] J.-M. Tacnet, M. Batton-Hubert, J. Dezert, A two-step fusion process for multi-criteria decision applied to natural hazards in mountains, Int. Workshop on Belief Functions, Brest, France, April 2010.

[20] J.-M. Tacnet, M. Rosalie, J. Dezert, E. Travaglini, Spatial information fusion: application to expertise and management of natural risks in mountains, in Proceedings of SAGEO'10 Int. Conf., Toulouse, France, pp.408-412, 17-19th November, 2010.

[21] J.-M. Tacnet, J. Dezert, Cautious OWA and Evidential Reasoning for Decision Making under Uncertainty, submitted to Proceedings of Fusion 2011 Int. Conf., Chicago, USA, July 5-8th, 2011.

[22] Y. M. Wang, J. B. Yang, and D. L. Xu, Environmental impact assessment using the evidential reasoning approach, European Journal of Operational Research, vol. 174, no. 3, pp. 1885-1913, 2006.

[23] L. Zadeh, Fuzzy sets, Information and Control, vol. 8, pp. 338-353, 1965.

[24] L. Zadeh, Fuzzy sets as a basis for a theory of possibility, Fuzzy Sets and Systems, vol. 1 , pp. 3-28, 1978. 